\title{
Lactobacillus gasseri as a Functional Food and Its Role in Obesity
}

\author{
Mohaddese Mahboubi ${ }^{*}$ \\ ${ }^{1}$ Medicinal Plants Research Department, Research and Development, Tabib Daru Pharmaceutical Company, Kashan, Iran
}

Corresponding Author: Mohaddese Mahboubi. PhD, Professor, Medicinal Plants Research Department, Research and Development, Tabib Daru Pharmaceutical Company, Kashan, Iran. Tel: +98-3155541000, Email: mahboubi1357@yahoo.com

Received December 10, 2018; Accepted April 18, 2019; Online Published June 15, 2019

\begin{abstract}
Obesity is a chronic, relapsing, progressive disease which is associated with a wide range of fatal diseases in the world. This review article evaluated the efficacy of Lactobacillus gasseri in the management of obesity. The information was extracted from electronic resources (PubMed, Science Direct, Wiley, and Google). The results of the research showed that the efficacy of $L$. gasseri on weight loss is strainsensitive. There are two well-known strains, BNR17 and SBT2055, which come from different sources and are used in many animal and clinical studies. More evidence on the efficacy of $L$. gasseri SBT2055 than on BNR17 is present in the literature. The daily dose of $L$. gasseri SBT2055 in clinical trials is higher than BNR17 at the dose of $10^{11}$. It seems that the use of a high dose of $L$. gasseri BNR17 in clinical trials is associated with more pleasant results on weight loss.

Keywords: Lactobacillus gasseri, Obesity, Weight Loss, Clinical studies, SBT2055, BNR17

Citation: Mahboubi M. Lactobacillus gasseri as a functional food and its role in obesity. Int J Med Rev. 2019;6(2):59-64. doi:10.29252/ IJMR-060206.
\end{abstract}

\section{Introduction}

Obesity is a chronic, relapsing, progressive disease. It is identified by abnormal and excessive fat accumulation which is defined as a body mass index (BMI) higher than $25 \mathrm{~kg} / \mathrm{m}^{2}{ }^{1}$ The health risks of obesity include a wide range of medical, surgical, gynecological, obstetric, social, and psychological conditions such as hypertension, cerebrovascular incidents, cancers, hyperlipidemia, coronary heart disease, diabetes mellitus, and degenerative arthritis. ${ }^{2}$ Therefore, the costs imposed by obesity are considerable. Changes in lifestyle, exercise, diet therapy, drugs with anti-lipase inhibitory effects, or medicinal plants are some ways to overcome obesity, but one critical factor related to obesity and metabolic disorders is the gut microbiota. The gut microbiota is related to obesity, type 2 diabetes, cardiovascular diseases, and non-alcoholic fatty liver disease. $^{3}$ The high proportion of intestinal firmicutes and low percentage of Bacteroides are associated with adipose tissue inflammation and obesity. ${ }^{4}$ Bacteroides have a lower number of enzyme genes for lipid and carbohydrate metabolism than firmicutes, and they reduce the energy uptake from diets. ${ }^{5}$ Probiotics as live healthy microorganisms are a promising approach to altering gut microbiota. ${ }^{6}$ Among probiotics, Lactobacillus gasseri is known as the weight loss probiotic. In this review article, the efficiency of L. gasseri in weight loss is discussed.

\section{Lactobacillus gasseri}

Lactobacillus gasseri is an autochthonous human intestinal bacterium which is acid resistant, bile tolerant, and distinguished from $L$. acidophilus by typical phenotype characteristics. The cell wall composition and electrophoretic mobility of $L$. gasseri $\mathrm{L}-\mathrm{LDH}$ is different from that of $L$. acidophillus. ${ }^{7}$ Cardiolipin as a membrane phospholipid of $L$. gasseri JCM1131 plays a pivotal role in the bile acid resistance of bacteria. ${ }^{8}$

Inulin, poly-oligosaccharides, and a broad range of fructooligosaccharides are synthesized by $L$. gasseri. ${ }^{9}$ Inulin is synthesized from sucrose using inulosucrase from $L$. gasseri DSM 20604. ${ }^{10}$

Lactobacillus gasseri reduces the number of fecal mutagenic enzymes, adheres to intestinal tissue, and stimulates macrophages in the intestine. It is able to adhere to Caco2 cells and exhibits antimicrobial activity. It reduced the fecal coliforms, serum total cholesterol, LDL-cholesterol, triglycerides, and bile acids in rats. The levels of acidic steroid increased in feces, but totally neutral steroids were not affected by $L$. gasseri in hypercholesterolemic rats. ${ }^{11}$

Two different mechanisms are proposed for reducing serum cholesterol using probiotic bacteria. L. gasseri is able to enhance the excretion of acidic steroids in feces and suppress the reabsorption of bile acids into the enterohepatic circulation. It is also able to adhere to the surface of the intestine and deconjugate bile acids with weak absorption from the small intestine is associated with high feces excretion by dietary fiber. Deconjucated bile acids are toxic for the growth of coliform or pathogenic bacteria. ${ }^{12}$ L. gasseri

Copyright $\odot 2019$ The Author(s). This is an open-access article distributed under the terms of the Creative Commons Attribution License (http:// creativecommons.org/licenses/by/4.0), which permits unrestricted use, distribution, and reproduction in any medium, provided the original work is properly cited. 
increases the small intestinal lipid metabolism by activating the long-chain acyl-CoA synthetase (ACSL3) pathway, which regulates whole-body glucose homeostasis in the body. ${ }^{13}$

The suppression of lipid absorption in the small intestine in the presence of L. gasseri has been reported. ${ }^{14}$ Inflammation in the intestine is correlated with obesity. Supplementing IL-10-deficient mice with L. gasseri (NC1501) for 4 weeks significantly reduced inflammation by producing MnSOD, which is associated with a reduction in the infiltration of neutrophils and macrophages. ${ }^{15}$ The immune-modulatory properties of $L$. gasseri have been confirmed. ${ }^{16}$

Lactobacillus gasseri SF1183 produces a molecule which interferes with the proliferation of human intestinal HCT116 cells and has anti-apoptotic activity and cytostatic effects on epithelial colon cells. The protective effect of $L$. gasseri against apoptosis of intestinal epithelial cells in the presence of inflammatory cytokines is caused by cell cycle arrest. ${ }^{17} \mathrm{~L}$. gasseri (SBT0270) increased the number of fecal Lactobacilli without affecting the number of fecal coliforms. Changing the gut's microbiota was associated with hypocholesterolemic effects (reductions in total cholesterol, HDL, LDL, and triglycerides) in hypercholesterolemic rats. Food intake was significantly decreased in the presence of L. gasseri ${ }^{18}$ L. gasseri ATCC 33323 exhibited protective effects against tetracyclineinduced body fat accumulation in a rat animal model. ${ }^{19}$

All evidence emphasized the efficiency of $L$. gasseri on the subject of obesity. Because the effect of probiotics in weight loss is species- and strain-specific and L. gasseri L66-5 promotes weight gain, ${ }^{20}$ the efficiency of the L. gasseri strain on weight loss was determined in detail.

\section{Lactobacillus gasseri SBT2055}

Animal Study on the Efficacy of Lactobacillus gasseri SBT2055 in Obesity

The administration of L. gasseri SBT2055 $\left(6 \times 10^{7} \mathrm{CFU} / \mathrm{g}\right)$ for 4 weeks in male Sprague-Dawley rats significantly decreased the average adipose size in mesenteric white adipose tissue $(P=0.004)$, serum leptin $(P=0.031)$, and liver triacylglycerol levels $(P=0.009)$; it further increased the number of small adipocytes from mesenteric $(P=0.004)$ and retroperitoneal $(P=0.053)$ adipose tissue. Neither adiponectin concentrations, serum glucose, serum and liver lipids, body and liver weight, nor adipose tissue were affected by a diet with $L$. gasseri. ${ }^{21} \mathrm{~A}$ significant reduction in adipocyte size in Sprague-Dawley rats was reported after the animals consumed a diet which included fermented skimmed milk (SM) containing L. gasseri SBT2055 $(6 \times 107 \mathrm{CFU} / \mathrm{g})$. Reductions in subcutaneous adipose tissue weight $(P<0.05)$, adipocyte size $(P<0.001)$, and serum leptin concentration $(P<0.05)$ in lean Zucker rats were observed after feeding them a diet containing $L$. gasseri for 4 weeks. L. gasseri had no effect on the mesenteric adipose tissue of obese Zucker rats, but the number of small adipocytes in the subcutaneous adipose tissue and lipoprotein lipase activity in adipose tissue of obese rats increased $(P<0.05)$. L. gasseri significantly reduced the concentration of serum leptin in both obese and lean Zucker rats, and it had no effect on serum glucose and adiponectin levels of Zucker rats. A significant reduction in serum and hepatic HDL-cholesterol and increases in the excretion of fecal fatty acids and total neutral fecal sterols were observed in both lean and obese Zucker rats. L. gasseri reduced the maximum transport rate of triacylglycerol, phospholipids, and lymphatic lipid absorption in the cannulated thoracic lymph ducts of Sprague-Dawley rats. ${ }^{14}$

Yogurt containing L. gasseri SBT2055 $\left(5 \times 10^{8}\right.$ CFU/g) consumed for 4 weeks inhibited the enlargement of visceral adipocytes and sICAMI as the inflammatory marker gene in obese male Sprague-Dawley rats. ${ }^{22}$ The body weight, fat tissue mass, and triglyceride levels of the liver were significantly decreased after feeding a $10 \%$ fat diet containing L. gasseri LG2055 $\left(2.5 \times 10^{8}\right)$ for 24 weeks to C57BL/6 mice. L. gasseri inhibited the pro-inflammatory genes in epididymal adipose tissue and liver lipogenic genes, improved the inflammatory condition in adipose tissue, and exhibited anti-obesity effects. ${ }^{23}$ Leptin expression and its release are closely linked with adipose size ${ }^{24}$; leptin regulates the fat synthesis and distributes and modulates hepatic $\beta$-oxidation, which inhibits hepatic triacylglycerol accumulation. Adipose tissue expansion is associated with an increase in fat cell size or cell number. ${ }^{25}$ Animal studies on the anti-obesity effects of L. gasseri SBT2055 have exhibited improvement in inflammatory conditions, a reduction in leptin, a reduction in the size of adipose cells and an increase in their number, and the excretion of fatty oil in the feces of animals, which help the anti-obesity effects of $L$. gasseri SBT2055; however, the colony-forming unit of cells is about $2.5 \times 10^{8}$ or lower, and performing animal studies with higher doses of L. gasseri SBT2055 is recommended.

Clinical Study on Efficacy of Lactobacillus gasseri SBT2055 in Obesity

Three clinical studies have investigated the efficacy of $L$. gasseri SBT2055 in treating obesity. The effects of $200 \mathrm{~g} / \mathrm{d}$ fermented milk containing $10^{11}$ CFU L. gasseri SBT2055 $(n=43)$, twice daily for 12 weeks on abdominal adiposity in adults with obese tendencies, a BMI of $24.2-30.7 \mathrm{~kg} / \mathrm{m}^{2}$, and abdominal visceral fat area of $81.2-17.8 .5 \mathrm{~cm}^{2}$ were compared with a placebo group $(\mathrm{n}=44)$. The results showed a significant reduction in abdominal visceral and subcutaneous fat areas $(4.6 \%$ and $3.3 \%)(P<00.01)$, body weight $(1.4 \%)(P<00.001)$, BMI (1.5\%), waist (1.8\%), and hip (1.5\%) circumferences for the intervention group compared with baseline. No changes in these parameters were observed for the placebo group. ${ }^{26}$ Another single blind controlled trial was performed on 20 Japanese hypertriacylglycerolemia subjects. Initially, the subjects took $200 \mathrm{~g} / \mathrm{d}$ fermented milk for 4 weeks (placebo group), and after a 4-week washout period, they received fermented milk containing $10^{11}$ CFU/day L. gasseri SBT2055. At the end of each week, 8-hour intake of oral fat loading test meals were fed. Blood samples were taken at fasting and every hour for 8 hours, and the postprandial serum non-estrified fatty acid, triacylglycerol, and fasting blood parameters were evaluated. The postprandial non-sterified fatty acid level was significantly reduced after consumption of yogurt containing 
L. gasseri LG2055. Anthropometric parameters (body weight, BMI, waist), insulin, glucose, triacylglycerol, total cholesterol, HDL, LDL, and liver enzymes (ALP, AST, ALT) showed no significant differences between the two groups. HbA1 levels were significantly greater in the probiotic group than in the placebo group. No adverse effects were observed during the study. The results of the study suggest its efficacy in reducing the risk of diseases such as obesity and type 2 diabetes. ${ }^{27}$ The study was limited by the number of volunteers enrolled in it.

A multi-center, double-blind, parallel-group randomized clinical trial was conducted on 210 healthy Japanese adults with large visceral fat areas $\left(80.2-187.8 \mathrm{~cm}^{2}\right)$. The subjects were randomly divided into three groups and fed $200 \mathrm{~g}$ of fermented milk containing $2 \times 10^{9}, 2 \times 10^{8}$, or 0 CFU L. gasseri SBT2055 for 12 weeks. The abdominal visceral fat areas changed $28.5 \%(P<0.01)$ and $28.2 \%(P<0.01)$ in the $2 \times 10^{9}$ and $2 \times 10^{8}$ groups, respectively. BMI, waist and hip circumferences were significantly reduced in both $L$. gasseri groups at weeks 8 and 12 from baseline, while no reduction was observed in the placebo group. There was a significant difference in BMI at week 12 in both probiotic groups compared with the placebo group. A significant reduction in fat mass was observed at week 12 from baseline in both probiotic groups compared with the control. No adverse events related to the consumption of probiotics were observed during the study. No physiologically significant changes or blood biochemical changes in triacylglycerol, total cholesterol, LDL cholesterol, HDL cholesterol, non-esterified fatty acids (NEFAs), total ketone bodies, lipoprotein, blood glucose, HbA1c, insulin, AST, ALT, ALP, L-lactate dehydrogenase, total protein, albumin, uric acid, blood urea $\mathrm{N}$, creatinine, $\mathrm{Na}, \mathrm{Cl}, \mathrm{K}, \mathrm{Ca}, \mathrm{P}$, $\mathrm{Mg}, \mathrm{Fe}$, leucocytes, erythrocytes, $\mathrm{Hb}$, hematocrit, or platelets were observed; all parameters were in normal ranges. ${ }^{28}$ The results of clinical studies showed that $2 \times 10^{11}$ L. gasseri SBT2055 from human fecal sources is a valuable probiotic bacterium for weight loss.

Mechanisms of Anti-obesity Effects of Lactobacillus gasseri SBT2055

Different mechanisms are involved in the anti-obesity effects of L. gasseri SBT2055. It has been confirmed that Ccl2 gene expression was inhibited by L. gasseri SBT2055 in dietinduced obese mice, which is associated with weight loss. $\mathrm{Ccl} 2$ gene express CC chemokine ligand 2, which infiltrates the macrophages into the adipose tissue. Administration of male mice C57BL/6J with normal, high-fat (control) and high-fat diets plus L. gasseri SBT2055 $\left(1 \times 10^{9} \mathrm{CFU} / \mathrm{g}\right)$ for 12 weeks, showed L. gasseri had no effects on abdominal fat weight and body weight, but L. gasseri significantly reduced the macrophages and the ratio of M1 macrophages as activated inflammatory macrophages to total macrophages in adipose tissues. The expression of Ccl2, Ccr2 and Lep genes were significantly reduced after the administration of L. gasseri SBT $2055 .{ }^{29}$ L. gasseri SBT 2055 for 21 weeks exerted anti-inflammatory and protective effects against intestinal barriers in high fat fed-C57BL/6 mice. Body weights and mesenteric and perirenal/retroperitoneal adipose tissues were significantly lower in the mice given L. gasseri SBT 2055 compared with the high fat-fed mice. L. gasseri SBT 2055 increased the number of M2 macrophages and decreased the M1:M2 ratio compared with the high fat-fed mice, which implies an improvement in an inflammatory condition of the body. A reduction in CD8+ $\mathrm{T}$ cells in adipose cells was observed after administration of L. gasseri LG2055. The permeability of the intestine in high fat-fed mice was reduced in the presence of L. gasseri, which improved the barrier functions, and these changes in gut permeability are related to adipose tissue inflammation. A reduction in intestine permeability is associated with a reduction in LPS or other inflammatory cytokines. ${ }^{30}$ IL-10 production is stimulated by dendritic cells in the presence of $L$. gasseri SBT2055 $5^{31}$ and partly increases the population of M2 macrophages in adipose tissue. ${ }^{30}$

The effects of L. gasseri SBT2055 on fat lipolysis was evaluated in vitro by evaluating the pancreatic lipase activity test. The effects of $100 \mathrm{~g}$ fermented milk containing L. gasseri $(\mathrm{n}=15)$ was compared with fermented milk without L. gasseri $\left(5 \times 10^{9} \mathrm{CFU}\right)(\mathrm{n}=15)$ on fecal fat excretions in healthy subjects (27-69 years old) in a double-blind, randomized, controlled clinical study. L. gasseri showed dose-dependent inhibitory effects against lipase activity and significantly increased the fecal fat concentration compared with the placebo group. L. gasseri suppressed lipid absorption in the small intestine. Triacylglycerol is hydrolyzed by pancreatic lipase in the duodenum. Therefore, inhibition of pancreatic lipase is the main strategy in controlling obesity. Direct inhibition of pancreatic lipase was not confirmed by L. gasseri LG2055, because it cannot use 4-MUO (methylumbelliferyl oleate) as a substrate. L. gasseri increased the fat droplet size which, along with specific surface area, can affect its digestion and absorption. By modifying fat emulsion properties, L. gasseri suppressed lipid digestion and absorption. The interaction between bile acid and L. gasseri SBT2055 increased the fat droplet size, which is associated with low absorption and digestion. BMI and total serum cholesterol concentration were significantly decreased in both the control and the active groups. $^{32}$

The protective effects of $L$. gasseri SBT2055 against oxidative stress in mammalian cells were confirmed. Up-regulation of the Nrf2 protein and its nuclear translocation activates the Nrf2-antioxidant response element (ARE) signaling pathway as the result of JNK activation in L. gasseri SBT2055-treated mammalian cells. The activation of the Nrf2-ARE pathway (JNK-dependent Nrf2-ARE signaling pathway) by L. gasseri strengthens the mammalian cells against oxidative stress. ${ }^{33}$ The lifespan of Caenorhabditis elegans was extended after feeding with L. gasseri LG2055 through activation of the Nrf2 ortholog, SKN-1, p38MAPK signaling pathway, and the defense system against oxidative stress. ${ }^{34}$

Oral administration of L. gasseri SBT2055 in mice increased the population of $\operatorname{Ig} \mathrm{A}^{+}$cells in Peyer's patch and in the lamina propria of the small intestine, which is associated with IgA production. A significant increase in $\operatorname{Ig} \mathrm{A}$ production was observed in a co-culture of B cells and dendritic cells from 
bone marrow. TLR2 signal is critical for IgA production. In addition, it has been demonstrated that L. gasseri SBT2055 stimulates BMDC to promote TGF- $\beta$, BAFF, IL-6, and IL-10 production, which are critical to the production of IgA from B-cells. IgA has a critical role in the host defense against mucosal pathogens. ${ }^{35}$ Its anti-inflammatory and antioxidant effects on fat droplet size, absorption, and digestion, and its inhibitory effects against pancreatic lipase are mechanisms that may be involved in the anti-obesity effects of L. gasseri SBT2055.

\section{Lactobacillus gasseri BNR17}

Animal Study on Efficacy of Lactobacillus gasseri BNR17 in Obesity

Lactobacillus gasseri BNR17 is isolated from human breast milk. L. gasseri BNR17 ( $\left.10^{9} \mathrm{CFU}\right)$, with no effect on daily food intake, reduced the percentage of increase in body weight $(P=0.0331)$, fat pad mass $(P<0.01)$, and white adipose tissue mass in rats fed a high-carbohydrate diet twice daily for 12 weeks. L. gasseri BNR17 had no significant effect on total cholesterol, HDL cholesterol, triglycerides, total protein, LDL cholesterol, kidney or spleen weight. L. gasseri reduced blood glucose levels and had protective effects against obesityinduced hepatomegaly through microvesicular steatosis, fibrosis, and sinusoidal dilation. ${ }^{36}$ The effects of $L$. gasseri $\operatorname{BNR} 17\left(10^{7}, 10^{8}, 10^{9}, 10^{10}\right)$ for 12 weeks in $\mathrm{db} / \mathrm{db}$ mice showed that the food intake, water intake, and amount of excrement decreased dose-dependently. Body weight was not reduced in the BNR17 groups. Fasting and postprandial blood glucose levels were significantly reduced after the administration of L. gasseri BNR17. An insignificant reduction in $\mathrm{HbA1c}$ was observed in the BNR17 groups. ${ }^{37}$ C57BL/6J mice supplemented with a high sucrose diet containing L. gasseri BNR17 $\left(10^{9}\right.$ or $\left.10^{10} \mathrm{CFU}\right)$ for 10 weeks showed a significant reduction in body weight, subcutaneous and abdominal fat mass, and white adipose tissue weight compared with mice on a normal diet. L. gasseri BNR17 is associated with increases in mRNA levels of fatty acid oxidation-related genes (ACO, CPT1, PPARa, PPARd), GLUT4 (main glucose transporter-4), and reductions in fatty acid synthesis-related genes (SREBP-1c, ACC), leptin, and insulin compared with the high-sucrose-diet group. ${ }^{38}$ The results of animal studies on the effects of L. gasseri BNR17 on obesity are promising but need more evaluation in clinical studies.

\section{Clinical Study on Efficacy of Lactobacillus gasseri BNR17 in Obesity \\ The efficacy of $L$. gasseri BNR17 ( $\mathrm{n}=23)$ on obese adults (19-60} years old) with a BMI higher than $23 \mathrm{~kg} / \mathrm{m}^{2}$ and a fasting blood sugar higher than $100 \mathrm{mg} / \mathrm{dL}$ for 12 weeks was compared with a placebo group of adults ( $n=29)$, and the body fat, body weight, and waist and hip circumferences were evaluated. Patients took 6 capsules of probiotic cells $\left(10^{10} \mathrm{CFU}\right), 3$ times a day. Although a slight reduction in body weight and a significant reduction in BMI, hip and waist circumferences were observed in the probiotic group compared with its baseline, the difference between the two groups was not significant. No significant differences were noted in fat or muscle amount,
BMI, visceral adipose tissue, or deep adipose tissue between the two groups after interventions. Vital symptoms including systolic and diastolic blood pressure and pulse rate showed no significant difference between the two groups. Fasting blood sugar, 2PPBS, HbA1c, insulin, total cholesterol, HDL, LDL, and triglyceride levels had no significant differences between the two groups. Blood chemistry parameters were not affected by the intervention. The only significant reduction observed was seen in hematocrit in the probiotic group $(P=0.02)$. No serious adverse effects were observed during either of the treatments. ${ }^{39}$

Lactobacillus gasseri BNR17 in low $\left(10^{9}\right.$ CFU/day) $(\mathrm{n}=30)$ and high doses $\left(10^{10} \mathrm{CFU} /\right.$ day $)(\mathrm{n}=30)$ was evaluated for its anti-obesity effects on 90 obese volunteers (20-75 years old) with BMIs of $25-35 \mathrm{~kg} / \mathrm{m}^{2}$ for 12 weeks in a randomized, double-blind, placebo controlled study. No adverse effects were recorded during the study, and the mean compliance ratio was $94 \%$. Anthropometric measures and demographic properties other than gender, waist circumference, and waistto-hip ratio were not significantly different among the three groups at baseline. Means of body weight, BMI, waist and hip circumferences had no significant difference after 12 weeks of treatment. The waist circumference was reduced and visceral adipose tissue was significantly reduced in the high probioticdose group. Biochemical parameters (total cholesterol, HDL, LDL, total glycerol, fasting blood glucose, insulin, leptin, adiponectin, CR) had no significant differences among the three groups. ${ }^{40}$ Although the daily dose of L. gasseri BNR17 was lower than that of L. gasseri SBT2055, the results on weight loss are hopeful.

\section{Conclusions}

The prevalence of obesity has been increasing in the world and is associated with many fatal diseases. Measures to prevent and treat obesity are vital. The side effects of drugs limit their applications; therefore, consumers prefer to use natural treatments, like medicinal plants and other sources. Probiotics are live, beneficial bacteria which have many functional properties in the body. L. gasseri is one well-known probiotic known for its weight loss properties. L. gasseri is used in many commercial, natural products for weight loss. In this review article, the use of two main strains, L. gasseri SBT2055 and BNR17, were discussed. The source of BNR17 is different from that of SBT2055. Although animal and clinical studies have been performed on both, there are more results and comprehensive studies on SBT2055. It is certain that the weight loss effects of L. gasseri are strain-sensitive, and the strain is very important in weight loss products. L. gasseri also improved inflammatory conditions in adipocyte tissue and the intestine, and helps the body resist oxidative stress. It increases fat droplet size and reduces their absorption and digestion. Furthermore, they deconjugate bile acids, which increases the excretion of fat and sterols in human feces. $L$. gasseri inhibits pancreatic lipase, reduces serum leptin, and increases the number of small adipocyte tissues in the body. It also inhibits the growth of adipocyte tissue in the body. By these mechanisms, specific strains of L. gasseri are good candidates for weight loss. 


\section{Conflict of Interest Disclosures}

The author declare that they have no conflicts of interest.

\section{References}

1. Muller MJ, Geisler C. Defining obesity as a disease. Eur J Clin Nutr. 2017;71(11):1256-1258.doi:10.1038/ejcn.2017.155.

2. Ogunbode AM, Fatiregun AA, Ogunbode OO. Health risks of obesity. Ann Ib Postgrad Med. 2009;7(2):22-25. doi:10.4314/ aipm.v7i2.64083.

3. Power SE, Fitzgerald GF, O'Toole PW, et al. Metabolic syndrome and obesity in adults. In: Guarino A, Quigley EMM, Walker WA, eds. Probiotic Bacteria and Their Effect on Human Health and Well-Being. Vol 107. Karger Publishers; 2013:103-121. doi:10.1159/000345750.

4. Million M, Maraninchi M, Henry M, et al. Obesity-associated gut microbiota is enriched in Lactobacillus reuteri and depleted in Bifidobacterium animalis and Methanobrevibacter smithii. Int J Obes (Lond). 2012;36(6):817-825. doi:10.1038/ijo.2011.153.

5. Million M, Lagier JC, Yahav D, Paul M. Gut bacterial microbiota and obesity. Clin Microbiol Infect. 2013;19(4):305-313. doi:10.1111/1469-0691.12172.

6. Mahboubi M, Kazempour N. The Effects of Inulin on Characteristics of Lactobacillus paracasei TD3 (IBRC-M 10784) as Probiotic Bacteria in vitro. Arch Iran Med. 2016;19(2):92-95.

7. Lauer E, Kandler O. Lactobacillus gasseri sp. nov., a new species of the subgenus Thermobacterium. Zentralblatt für Bakteriologie: I. Abt. Originale C: Allgemeine, angewandte und ökologische Mikrobiologie. 1980;1(1):75-78. doi:10.1016/S01725564(80)80019-4.

8. Kato $\mathrm{S}$, Tobe $\mathrm{H}$, Matsubara $\mathrm{H}$, et al. The membrane phospholipid cardiolipin plays a pivotal role in bile acid adaptation by Lactobacillus gasseri JCM1131(T). Biochim Biophys Acta Mol Cell Biol Lipids. 2019;1864(3):403-412. doi:10.1016/j. bbalip.2018.06.004.

9. Anwar MA, Kralj S, Pique AV, Leemhuis H, van der Maarel MJ, Dijkhuizen L. Inulin and levan synthesis by probiotic Lactobacillus gasseri strains: characterization of three novel fructansucrase enzymes and their fructan products. Microbiology. 2010;156(Pt 4):1264-1274. doi:10.1099/mic.0.036616-0.

10. Ni D, Zhu Y, Xu W, Bai Y, Zhang T, Mu W. Biosynthesis of inulin from sucrose using inulosucrase from Lactobacillus gasseri DSM 20604. Int J Biol Macromol. 2018;109:1209-1218. doi:10.1016/j. ijbiomac.2017.11.120.

11. Usman, Hosono A. Bile tolerance, taurocholate deconjugation, and binding of cholesterol by Lactobacillus gasseri strains. J Dairy Sci. 1999;82(2):243-248.doi:10.3168/jds.S0022-0302(99)75229-X.

12. Usman, Hosono A. Effect of administration of Lactobacillus gasseri on serum lipids and fecal steroids in hypercholesterolemic rats. J Dairy Sci. 2000;83(8):1705-1711. doi:10.3168/jds.S00220302(00)75039-9.

13. Bauer PV, Duca FA, Waise TMZ, et al. Lactobacillus gasseri in the upper small intestine impacts an ACSL3-dependent fatty acidsensing pathway regulating whole-body glucose homeostasis. Cell Metab. 2018;27(3):572-587.e576. doi:10.1016/j. cmet.2018.01.013.

14. Hamad EM, Sato M, Uzu K, et al. Milk fermented by Lactobacillus gasseri SBT2055 influences adipocyte size via inhibition of dietary fat absorption in Zucker rats. Br J Nutr. 2009;101(5):716-724. doi:10.1017/s0007114508043808.

15. Carroll IM, Andrus JM, Bruno-Barcena JM, Klaenhammer TR, Hassan HM, Threadgill DS. Anti-inflammatory properties of Lactobacillus gasseri expressing manganese superoxide dismutase using the interleukin 10-deficient mouse model of colitis. Am J Physiol Gastrointest Liver Physiol. 2007;293(4):G729-738. doi:10.1152/ajpgi.00132.2007.

16. Morita H, He F, Kawase M, et al. Preliminary human study for possible alteration of serum immunoglobulin $\mathrm{E}$ production in perennial allergic rhinitis with fermented milk prepared with Lactobacillus gasseri TMC0356. Microbiol Immunol. 2006;50(9):701-706. doi:10.1111/j.1348-0421.2006.tb03842.x.

17. Di Luccia B, Manzo N, Baccigalupi L, et al. Lactobacillus gasseri SF1183 affects intestinal epithelial cell survival and growth. PLoS One. 2013;8(7):e69102. doi:10.1371/journal.pone.0069102.

18. Usman, Hosono A. Hypocholesterolemic effect of Lactobacillus gasseri SBT0270 in rats fed a cholesterol-enriched diet. J Dairy Res. 2001;68(4):617-624. doi:10.1017/S0022029901005179.

19. Marciano JJ, de Sa Del Fiol F, Ferreira ACMT, Marques MC, Santana LL. Changes in weight and body fat after use of tetracycline and Lactobacillus gasseri in rats. Braz J Pharm Sci. 2017;53(1):e16059. doi:10.1590/s2175-97902017000116059.

20. Rouxinol-Dias AL, Pinto AR, Janeiro C, et al. Probiotics for the control of obesity-Its effect on weight change. Porto Biomed J. 2016;1(1):12-24. doi:10.1016/j.pbj.2016.03.005.

21. Sato M, Uzu K, Yoshida T, et al. Effects of milk fermented by Lactobacillus gasseri SBT2055 on adipocyte size in rats. Br J Nutr. 2008;99(5):1013-1017. doi:10.1017/s0007114507839006.

22. Kadooka Y, Ogawa A, Ikuyama K, Sato M. The probiotic Lactobacillus gasseri SBT2055 inhibits enlargement of visceral adipocytes and upregulation of serum soluble adhesion molecule (sICAM-1) in rats. Int Dairy J. 2011;21(9):623-627. doi:10.1016/j. idairyj.2011.02.001.

23. Miyoshi M, Ogawa A, Higurashi S, Kadooka Y. Anti-obesity effect of Lactobacillus gasseri SBT2055 accompanied by inhibition of pro-inflammatory gene expression in the visceral adipose tissue in diet-induced obese mice. Eur J Nutr. 2014;53(2):599-606. doi:10.1007/s00394-013-0568-9.

24. Lonnqvist F, Nordfors L, Jansson M, Thorne A, Schalling M, Arner $P$. Leptin secretion from adipose tissue in women. Relationship to plasma levels and gene expression. J Clin Invest. 1997;99(10):23982404. doi:10.1172/jci119422.

25. Cohen P, Friedman JM. Leptin and the control of metabolism: role for stearoyl-CoA desaturase-1 (SCD-1). J Nutr. 2004;134(9):2455s-2463s. doi:10.1093/jn/134.9.2455S.

26. Kadooka Y, Sato M, Imaizumi K, et al. Regulation of abdominal adiposity by probiotics (Lactobacillus gasseri SBT2055) in adults with obese tendencies in a randomized controlled trial. Eur J Clin Nutr. 2010;64(6):636-643. doi:10.1038/ejcn.2010.19.

27. Ogawa A, Kadooka Y, Kato K, Shirouchi B, Sato M. Lactobacillus gasseri SBT2055 reduces postprandial and fasting serum nonesterified fatty acid levels in Japanese hypertriacylglycerolemic subjects. Lipids Health Dis. 2014;13:36. doi:10.1186/1476511x-13-36.

28. Kadooka Y, Sato M, Ogawa A, et al. Effect of Lactobacillus gasseri SBT2055 in fermented milk on abdominal adiposity in adults in a randomised controlled trial. Br J Nutr. 2013;110(9):1696-1703. doi:10.1017/s0007114513001037.

29. Ukibe K, Miyoshi M, Kadooka Y. Administration of Lactobacillus gasseri SBT2055 suppresses macrophage infiltration into adipose tissue in diet-induced obese mice. Br J Nutr. 2015;114(8):11801187. doi:10.1017/s0007114515002627.

30. Kawano M, Miyoshi M, Ogawa A, Sakai F, Kadooka Y. Lactobacillus gasseri SBT2055 inhibits adipose tissue inflammation and intestinal permeability in mice fed a high-fat diet. J Nutr Sci. 2016;5:e23. doi:10.1017/jns.2016.12.

31. Sakai F, Hosoya T, Ono-Ohmachi A, et al. Lactobacillus gasseri SBT2055 induces TGF-beta expression in dendritic cells and activates TLR2 signal to produce IgA in the small intestine. PLoS One. 2014;9(8):e105370. doi:10.1371/journal.pone.0105370.

32. Ogawa A, Kobayashi T, Sakai F, Kadooka Y, Kawasaki Y. Lactobacillus gasseri SBT2055 suppresses fatty acid release through enlargement of fat emulsion size in vitro and promotes fecal fat excretion in healthy Japanese subjects. Lipids Health Dis. 2015;14:20. doi:10.1186/s12944-015-0019-0.

33. Kobatake E, Nakagawa H, Seki T, Miyazaki T. Protective effects and functional mechanisms of Lactobacillus gasseri SBT2055 against 
oxidative stress. PLoS One. 2017;12(5):e0177106. doi:10.1371/ journal.pone.0177106.

34. Nakagawa $\mathrm{H}$, Shiozaki T, Kobatake E, et al. Effects and mechanisms of prolongevity induced by Lactobacillus gasseri SBT2055 in Caenorhabditis elegans. Aging Cell. 2016;15(2):227-236. doi:10.1111/acel.12431.

35. Sakai F, Hosoya T, Ono-Ohmachi A, et al. Lactobacillus gasseri SBT2055 induces TGF-beta expression in dendritic cells and activates TLR2 signal to produce IgA in the small intestine. PLoS One. 2014;9(8):e105370. doi:10.1371/journal.pone.0105370.

36. Kang JH, Yun SI, Park HO. Effects of Lactobacillus gasseri BNR17 on body weight and adipose tissue mass in diet-induced overweight rats. J Microbiol. 2010;48(5):712-714. doi:10.1007/s12275-0100363-8.

37. Yun SI, Park HO, Kang JH. Effect of Lactobacillus gasseri BNR17 on blood glucose levels and body weight in a mouse model of type 2 diabetes. J Appl Microbiol. 2009;107(5):1681-1686. doi:10.1111/ j.1365-2672.2009.04350.x.

38. Kang JH, Yun SI, Park MH, Park JH, Jeong SY, Park HO. Anti-obesity effect of Lactobacillus gasseri BNR17 in high-sucrose diet-induced obese mice. PLoS One. 2013;8(1):e54617. doi:10.1371/journal. pone.0054617.

39. Jung SP, Lee KM, Kang JH, et al. Effect of Lactobacillus gasseri BNR17 on overweight and obese adults: a randomized, doubleblind clinical trial. Korean J Fam Med. 2013;34(2):80-89. doi:10.4082/kjfm.2013.34.2.80.

40. Kim J, Yun JM, Kim MK, Kwon O, Cho B. Lactobacillus gasseri BNR17 Supplementation Reduces the Visceral Fat Accumulation and Waist Circumference in Obese Adults: A Randomized, DoubleBlind, Placebo-Controlled Trial. J Med Food. 2018;21(5):454-461. doi:10.1089/jmf.2017.3937. 